\title{
Adaptação acadêmica de estudantes cotistas e não cotistas
}

\author{
Marcia Cristina Monteiro ${ }^{1}$ \\ Adriana Benevides Soares \\ Universidade Salgado de Oliveira, Rio de Janeiro-RJ, Brasil
}

\section{Resumo}

A adaptação acadêmica é um fenômeno multideterminado e supõe-se que haja diferença no ajustamento dos alunos que ingressam pelo sistema de cotas daqueles que ingressam pelo caminho tradicional. O objetivo foi investigar o impacto das variáveis Habilidades Sociais, Resolução de Problemas Sociais e Coping na Adaptação Acadêmica de estudantes cotistas e não cotistas. Participaram 637 estudantes e a coleta de dados se deu pela aplicação de instrumentos relativos aos construtos e utilizou-se equações estruturais. Os resultados sugerem que o estudante cotista confronta as adversidades, procura suporte para superá-las e acredita ser capaz de se organizar e concluir com sucesso a graduação. O estudo contribuiu com o conhecimento sobre variáveis que impactam na adaptação acadêmica. Pesquisas prospectivas podem considerar dados sociodemográficos.

Palavras-chave: Adaptação, universitários, ensino superior

\section{Abstract: Academic adaptation of quota and non-quota students}

Academic adaptation is a multi-determined phenomenon and it is assumed that there is a difference in the adjustment process of students enrolled by the quota system of those enrolled by the traditional way. This study aimed to investigate the impact of the variables Social Skills, Social Problem Solving and Coping on Academic Adaptation of quota and non-quota students. A total of 637 students participated and the data collection was carried out by the application of instruments related to the constructs and used structural equations. The results suggest that the quota student confronts adversities, seeks support to overcome them and believes he or she is able to organize and successfully complete the course. The study contributed with the knowledge of variables that impact on academic adaptation. Prospective studies may consider sociodemographic data.

Keywords: Adaptation, College students, higher education

\section{Resumen: Adaptación académica de estudiantes con y sin cuota}

La adaptación académica es un fenómeno multideterminado y se supone que existen diferencias en el proceso de adaptación entre los alumnos que ingresan a la universidad por el sistema de cuotas y aquellos que acceden por el sistema tradicional. El objetivo del estudio fue investigar el impacto de las variables Habilidades Sociales, Resolución de Problemas Sociales y Afrontamiento en la Adaptación Académica de estudiantes con cuota y sin cuota. Participaron 637 estudiantes y la recolección de datos se realizó mediante la aplicación de instrumentos relativos a los constructos, utilizando ecuaciones estructurales. Los resultados indican que el estudiante que ingresa por el sistema de cuotas enfrenta adversidades, busca apoyo para superarlas y considera ser capaz de organizarse académicamente y terminar exitosamente la carrera universitaria. El estudio aportó al conocimiento sobre el impacto de variables que inciden en la adaptación académica. Estudios prospectivos pueden considerar datos sociodemográficos.

Palabras clave: Adaptación, universidad, educación superior

\footnotetext{
${ }^{1}$ Endereço para correspondência: Avenida Marechal Deodoro, 217, Centro, 24030-60, Niterói, RJ.E-mail: marcialauriapsi@outlook.com
} 
$\mathrm{O}$ ingresso na universidade implica em investimento pessoal, material e social. Muitos alunos dedicam os anos do Ensino Médio para obterem coeficientes de rendimento que lhes confira valores de competitividade ao se submeterem aos concursos de seleção para o Ensino Superior. Outros estudantes buscam esse preparo em cursos particulares ou comunitários, o que requer investimento financeiro, e há aqueles que abdicam, em muitos casos, de horas de lazer para se dedicarem aos estudos. Enfim, é uma etapa da vida que exige empenho e seriedade nas metas a serem alcançadas (Moreno \& Soares, 2014).

Em paralelo a este investimento pessoal, estão as metas oficiais do país para o Ensino Superior. A Lei 13.005/14 - Plano Nacional de Educação (PNE) - para o decênio de 2014 a 2024 objetiva a ampliação da escolaridade média da população brasileira, a interiorização das instituições de Ensino Superior, o aumento no número de vagas nos cursos de graduação, além de implementar políticas afirmativas para a população historicamente afastada desta modalidade de ensino. Segundo a Organização de Cooperação e Desenvolvimento Econômico (OCDE; 2012), a Educação Superior tem o papel de promover a democracia, os direitos sociais e o desenvolvimento dos processos cognitivos e interpessoais. Entretanto, a OCDE (2012) lembra que a universidade precisa estar atenta à qualidade do ensino e à aprendizagem oferecidas e que os alunos são o centro dessa atenção, considerando ainda as diversidades acadêmicas, sociais e culturais.

Castro e Almeida (2016) afirmam que a garantia de um projeto educacional de qualidade passa por um conjunto de aspectos que dizem respeito à própria instituição, às políticas governamentais de acesso, aos ambientes de aprendizagem, à metodologia de ensino, aos processos de avaliação e aos aspectos inerentes aos graduandos. Os autores apontam que o aluno é o principal agente do processo de formação e sucesso profissional e que as contingências que antecedem a entrada do aluno na universidade, as características sociodemográficas, a opção de curso, as condições de acesso, assim como o bem-estar, a autonomia e a satisfação do estudante também estão relacionadas à adaptação ao Ensino Superior.

A democratização do ingresso na Educação Superior não garante a permanência ou mesmo a qualidade dos processos de aprendizagem (Matta, Lebrão, \& Heleno, 2017). O estudante que tem chegado à graduação traz uma diversidade de experiências acadêmicas, necessidades e expectativas iniciais que desafiam as instituições no aprimoramento dos projetos pedagógicos, além de sinalizar à complexidade e multifatorialidade das questões que envolvem a adaptação à universidade (Almeida, 2007; Araújo, 2017; Souza, Lourenço, \& Santos, 2016). Souza et al. (2016) destacam que na universidade quanto mais o estudante sabe o que pretende, investe e se organiza para obter o que almeja, demonstra comprometimento com o curso e com a futura carreira. Desta forma, procura participar de atividades extracurriculares, de projetos de pesquisas e monitoria, se permite vivenciar aprendizados e identificar diferentes campos de atuação e compreender a variabilidade do mercado de trabalho.

Ao longo de algumas décadas, modelos teóricos tentam explicar o fenômeno de interação à universidade. Tinto (1975) propôs um modelo sobre a integração acadêmica e social do aluno para adaptação, sucesso na graduação e na carreira. Para o autor, a integração acadêmica e social se relaciona ao fato do estudante permanecer ou abandonar o curso e tem relação com a evasão observada no Ensino Superior. Ademais, o conceito de integração refere-se ao fato do aluno inserir- se no novo contexto, considerando as características e dinâmicas existentes a serem vivenciadas, como também regular o desempenho, apreciar os conteúdos curriculares, compreender que todas as disciplinas têm destaque na formação e conhecer as diretrizes do curso. O segundo conceito, integração social, refere-se à satisfação de compartilhar novas relações interpessoais com todos aqueles que compõem a comunidade universitária, participar de atividades curriculares e extracurriculares que propiciem o aprendizado e os novos vínculos. A associação positiva com a instituição e o propósito de concluir o curso são outras variáveis pertencentes ao modelo.

O modelo teórico proposto por Almeida (2007) para adaptação acadêmica se diferencia de Tinto (1975) por destacar a relação com as competências do discente, docente e as características do contexto universitário. No que se refere as competências do aluno, o autor destaca os conhecimentos trazidos da formação secundária, os quais em muito contribuem para o rendimento escolar, assim como as capacidades cognitivas e intelectuais que colaboram no processamento da quantidade e complexidade das informações. Ademais, as percepções que o estudante possui das próprias capacidades e rendimento, como a capacidade de criticar e analisar, o envolvimento nos estudos e a autorregulação do comportamento podem promover a organização de pensamentos, sentimentos e ações planejadas e sistemáticas, que incrementam a motivação, a aprendizagem e consequentemente a integração acadêmica e social.

Em relação aos docentes, Almeida (2007) ressalta que as competências pedagógicas e as qualidades científicas auxiliam nas escolhas de estratégias de ensino mais diversificadas, as quais, supostamente, contribuem para o desenvolvimento da cooperação, da interação e da resolução de situações. Finalmente, o contexto de ensino e de 
aprendizagem possibilita uma maior adaptação ao Ensino Superior por meio da flexibilização curricular, da própria infraestrutura oferecida e pelo tipo de curso. $\mathrm{O}$ autor ressalta que a formação acadêmica não se inicia ou mesmo termina no espaço universitário, mas é um dos elementos deste processo e que deverá dialogar com o mundo profissional/trabalho.

Neste contexto, compreende-se que pode haver diferença no processo de adaptação daqueles alunos que ingressam pelo sistema de cotas em relação aos não cotistas. As cotas fazem parte de um conjunto de ações afirmativas que pretendem atenuar as desigualdades em uma sociedade. Em termos de Brasil, em 2002 é promulgada a Lei Federal 10.558 também denominada de "Lei das cotas" que cria o Programa Diversidade na Universidade e estabelece outras providências e conta com decretos, como o 4876/2003 (alterado pelo decreto 5193/2004) que trata da análise, seleção e aprovação de projetos inovadores de graduações, financiamento e transferências, concessão de bolsas de manutenção e prêmios (Cardoso, 2008). Segundo Ribeiro, Amaral, Peixoto, Oliveira e Bastos (2014) as universidades públicas vêm aderindo paulatinamente, desde 2003, às ações afirmativas. Os autores ainda ressaltam que com a aprovação da 12.711/12 houve a garantia de reserva de 50\% de vagas nas Instituições de Ensino Superior públicas (IES) Federais de cotas raciais e sociais até 2016, com revisão do programa em 2022. Entretanto, pesquisas apontam para pré-concepções que alunos cotistas e não cotistas apresentam sobre as cotas enquanto ações afirmativas e mesmo referentes as habilidades cognitivas, intelectuais, relacionais e escolares antecedentes dos alunos cotistas (Santos, 2013).

Santos (2013) pesquisou as diferenças nas vivências acadêmicas de estudantes cotistas e não cotistas e comparou o impacto das variáveis integração social e acadêmica quanto à satisfação com a formação e intenção de evasão destes estudantes. $\mathrm{O}$ estudo contou com amostra de 3.137 estudantes da Universidade Federal da Bahia, sendo que 1.771 eram alunos cotistas. Foi utilizada a Escala de Ajustamento ao Ensino Superior e os resultados obtidos indicaram que, para os estudantes cotistas, a satisfação com a graduação assim como a questões vinculadas a escolha da carreira e a inserção no mundo do trabalho predizem a evasão.

Schofield, Hausmann, Ye e Woods (2010) estudaram a relação de amizade entre pares intergrupais de uma universidade norte-americana de maioria branca, partindo do pressuposto que as relações intergrupais contribuem para a diminuição de situações prejudiciais ao aluno, implementam a empatia externa do grupo e diminui a ansiedade intergrupal. A pesquisa contou com estudantes ingressantes, de ambos os sexos, sendo 1.200 de brancos norte-americanos e 145 norte-americanos de descendência africana. Os autores destacam que os relacionamentos de amizade intergrupais se intensificaram ao final do primeiro ano e a relação de amizade intergrupo está associada as vivências estudantis anteriores diretas ou indiretas e na própria universidade, com a oportunidade de ter colegas de quarto ou de grupo de atividades intergrupais.

Diante da complexidade da adaptação ao Ensino Superior e das possíveis questões que são geradas a partir das dificuldades no ajustamento, é mister investigar um conjunto de variáveis que podem contribuir para a compreensão e possíveis ações de intervenção. Estar em um novo ambiente estudantil, diante de novos desafios, resolvendo situações problemas no contexto universitário é uma tarefa que envolve as relações interpessoais. A diversidade das relações no ambiente estudantil, exige dos estudantes e futuros profissionais a habilidade de ser capaz de resolver com precisão e rapidez as situações enfrentadas. Estudantes com habilidades de resolução de problemas sociais estabelecem relações interpessoais com pares, resolvendo impasses com colegas, professores e gestores. Esta habilidade também deveria ser aprendida no contexto universitário e ter continuidade na futura carreira (Fang, Luo, Li, \& Huang, 2016). Outrossim, os autores destacam que a resolução de problemas sociais está ainda relacionada ao bem-estar, a autoestima, a satisfação, as habilidades sociais e a competência interpessoal.

A resolução de problemas sociais diz respeito a habilidade de resolver problemas reais, do mundo real. É definida como um processo autodirecionado, cognitivo e comportamental, no qual o sujeito tenta descobrir maneiras integradas ou efetivas para enfrentar situações problemáticas do cotidiano (D'Zurilla \& Sheedy, 1992; D'Zurilla, Nezu, \& Maydeu-Olivares, 2002).

A relevância da resolução de problemas sociais é apontada em pesquisa com estudantes do Ensino Superior realizado por Lau (2014). A pesquisa contou com uma amostra de 194 graduandos de enfermagem da universidade pública de Macau (China) e teve como objetivo identificar a habilidade de resolução de problemas sociais e a associação da variável resolução de problemas sociais com a habilidade de comunicação, interação na clínica e disfunção interpessoal. Para tal, o autor utilizou questionário Sóciodemográfico, Inventário de Resoluções de Problemas Sociais na forma reduzida e adaptada para a realidade chinesa, Communication Ability Scale, Clinical Interactive Scale e Interpersonal Dysfunction Checklist. $\mathrm{O}$ autor concluiu que as variáveis investigadas apresentaram correlação com a habilidade de resolução de problemas sociais e sugere que seja implementado no currículo 
acadêmico o treinamento em resolução de problemas sociais, considerando que a carreira de enfermagem requer essa habilidade para o efetivo cuidado do paciente.

Fang et al. (2016), em estudo sobre a habilidade de resolução de problemas em estudantes de enfermagem, contaram com uma amostra de 681 participantes de universidades chinesas e utilizaram o Inventário de Resolução de Problemas Sociais na versão reduzida e adaptada para a realidade local. Os estudantes finalistas apresentam escores mais elevados no fator Habilidades Positivas de Resolução de Problemas Sociais e alunos que foram submetidos a treinamento autodirecionado em problemas apresentaram escores elevados em Orientação Positiva para o Problema. O grupo com treinamento específico em Pensamento Crítico exibiu resultados negativos de resolução de problemas comparados ao grupo sem treinamento. Os autores concluíram que as habilidades de resolução de problemas sociais variam conforme as estratégias empregadas no processo de ensino e aprendizagem e sugerem que a aprendizagem autodirigida e baseada em problemas pode ser efetiva para aprimorar a habilidade de resolução de problemas sociais.

Neste sentido, além da variável resolução de problemas sociais promover a integração do estudante no contexto universitário, pesquisas apontam que as habilidades sociais também estão associadas à adaptação acadêmica. As habilidades sociais possibilitam um maior envolvimento nas atividades acadêmicas, engajamento na nova modalidade de ensino e sucesso nas metas profissionais (Couto, Vandenberghe, Tavares, \& Silva, 2012; Soares, G. Gomes, Maia, Gomes, \& Monteiro, 2016; Soares \& Del Prette, 2015; Soares, Poubel, \& Melo, 2009).

Define-se habilidades sociais como "classes de comportamentos sociais que somente podem ser classificadas como tais na medida em que contribuem para a competência social" (A. Del Prette \& Del Prette, 2017, p. 21). A Educação Superior deve primar pelo desenvolvimento de habilidades cognitivas e metacognitivas, pela capacitação teórico-técnica e pela competência social associada a desempenhos sociais adequados. Em tese, os cursos de graduação preparam para a formação teórica e técnica. Entretanto, as competências sociais ficam a cargo do estudante, que muitas vezes não possuem ainda recursos próprios para desenvolvê-las (Mello \& Soares, 2014). As habilidades sociais são demandadas nas relações interpessoais. Nos primeiros períodos na universidade os estudantes são mais exigidos em termos de novos vínculos com um número grande de pessoas desconhecidas e lidam com exigências frequentes (Seco, 2005). As mudanças sociais promovidas pelo ambiente acadêmico vão além da aquisição de conhecimentos e da adaptação ao novo contexto, mas requerem competência acadêmica, estando a competência acadêmica relacionada à competência social (Soares et al., 2009).

Estudo desenvolvido por Iturra, Goic, Astete e Jara (2012) apontaram a diferença entre gêneros, habilidades sociais e rendimento acadêmico. Com uma amostra de 200 estudantes universitários, de diferentes graduações, do segundo e terceiros períodos de uma universidade chilena, por meio do Inventário de Comportamento Interpessoal e das médias totais de rendimento das diferentes graduações, os autores destacaram que os homens são mais assertivos em relação ao sexo oposto e rendimento acadêmico. Em outros termos, o aumento de comportamentos assertivos nos vínculos amorosos com as parceiras implica no aumento do rendimento e vice-versa. Por outro lado, as mulheres apresentam correlação entre habilidades assertivas com o aumento do rendimento acadêmico e indicadores de agressividade se correlacionam com a diminuição do desempenho acadêmico. Habilidades de cooperação e colaboração com os pares se associam com o ambiente acadêmico pelo fato de desenvolver habilidades relacionais tendo impacto no rendimento acadêmico.

Em estudo de Soares e Del Prette (2015), as autoras sugerem que um bom repertório de habilidades sociais contribui com a qualidade das interações na universidade. As habilidades sociais, segundo as autoras, facilitam a adaptação do estudante ao Ensino Superior, considerando que as aprendizagens que ocorrem na Educação Superior são cognitivas e relacionais, o que auxiliaria no desenvolvimento de habilidades sociais tanto para o contexto universitário, como profissional e para própria vida.

O contexto acadêmico pode representar para muitos estudantes um conjunto de situações de difícil manejo. O estudante lida com reponsabilidades constantes e diversas, como exposição e realização de trabalhos, exigências familiares, sociais e econômicas (Sicre \& Casaro, 2014). O coping é um conjunto de estratégias adaptativas utilizadas pelo sujeito para se ajustar a situações adversas ou mesmo estressantes (Lazarus \& Folkman, 1984; Antoniazzi, Dell'Aglio, \& Bandeira, 1998). Para Mofidi, El-alayli e Brown (2014); Oliveira, Carlotto, Vasconcelos e Dias (2014); Karnal, Monteiro, A. Santos e Santos (2017), pesquisas têm dedicado menos atenção aos fatores psicológicos internos, protetivos e as estratégias de coping que dizem respeito a adaptação acadêmica e que em muitas situações podem promover a retenção. Para os autores, estilos adaptativos de coping estão associados à integração acadêmica e social, ao aumento do interesse, da persistência e o compromisso com a instituição.

Sicre e Casaro (2014) realizaram pesquisa sobre os estilos de estratégias de coping em estudantes universitários e a relação com variáveis como o ano cursado, gênero 
e aprovação nos componentes curriculares. Participaram 140 alunos do curso de Psicologia de uma universidade particular da Argentina e utilizaram o Inventário de Respostas de Enfrentamento para jovens adaptado para a realidade local. O estudo revelou que o fator Enfrentamento por Aproximação Cognitiva foi a estratégia mais utilizada pelos estudantes. Esta refere-se aos esforços cognitivos para compreender e se preparar mentalmente para enfrentar elementos geradores de estresse e as respectivas consequências. A relação entre o fator Enfrentamento por Aproximação Cognitiva e a quantidade de disciplinas foi considerada fraca e positiva e o estudo não encontrou diferença significativa entre gêneros.

A adaptação acadêmica não é um bloco único e homogêneo, mas é composta por diferentes variáveis que precisam ser pesquisadas e mensuradas. Desta forma, considerando os múltiplos aspectos que estão associados ao ajustamento do estudante ao Ensino Superior, o objetivo do estudo é investigar o impacto das variáveis Coping, Habilidades Sociais, Resolução de Problemas Sociais, sobre a Adaptação Acadêmica de estudantes cotistas e não cotistas das Instituições de Ensino Superior públicas.

\section{Método}

\section{Participantes}

O estudo contou com uma amostra de 315 estudantes de IES pública, advindos de diferentes graduações, períodos, de ambos os sexos, sendo 115 (36,5\%) homens e com idade variando entre 18 a 38 anos $(M=24,7 ; D P$ $=6,3)$. Em termos de períodos participaram 87 (27,6\%) alunos do primeiro período, $55(17,4 \%)$ do segundo, 32 $(10,2 \%)$ do terceiro, $23(7,3 \%)$ do quarto, $32(10,2 \%)$ do quinto, $35(11,1 \%)$ do sexto, $23(7,6 \%)$ do sétimo, nove $(2,8 \%)$ do oitavo, cinco $(1,5 \%)$ do nono, oito $(2,5 \%)$ do décimo, três $(0,9 \%)$ do décimo primeiro, dois $(0,6 \%)$ do décimo segundo e um $(0,3 \%)$ do décimo terceiro. Os níveis socioeconômicos foram $25(7,9 \%)$ participantes pertencentes à classe $\mathrm{A}, 24(7,6 \%)$ à $\mathrm{B} 1,97(30,8 \%)$ à $\mathrm{B} 2,115(36,5 \%)$ à $\mathrm{C} 1,50(15,9 \%)$ à $\mathrm{C} 2$ e $4(1,3 \%)$ à DE (Associação Brasileira das Empresas de Pesquisa, 2015). Em termos de graduações, foram $76(24,1 \%)$ de Pedagogia, 45 (14,3\%) de Física, 33 (10,5\%) de Turismo, 33 (10,5\%) de Letras, 25 (8\%) de Psicologia, 16 (5\%) para Direito, $16(5 \%)$ para outros cursos respectivamente, 13 $(4,2 \%)$ de Biologia, 12 (3,7\%) de História, nove $(2,8 \%)$ de Biomedicina, oito $(2,5 \%)$ para Matemática, Enfermagem foram oito $(2,5 \%)$, cinco $(1,7 \%)$ de Geografia, quatro $(1,2 \%)$ de Engenharia, três $(0,9 \%)$ de Química, dois $(0,7 \%)$ de Sistema da Informação, dois $(0,9 \%)$ de Comunicação
Social, um $(0,4 \%)$ para o curso de Filosofia e um $(0,4 \%)$ para Estatística, Música e Sociologia.

$\mathrm{Na}$ amostra total contou-se com 180 estudantes cotistas, sendo 136 mulheres e 44 homens. Para os cotistas foram 71 (22,5\%) alunos da graduação de Pedagogia, 25 (7,9\%) de Psicologia, 23 (7,3\%) de Letras, 13 (4,12\%) de Biologia, oito $(2,5 \%)$ de Enfermagem, sete $(2,2 \%)$ de Matemática, cinco $(1,6 \%)$ de Turismo, quatro $(1,2 \%)$ de Engenharia, três $(0,95 \%)$ de outros cursos, dois $(0,63 \%)$ de Sistema da Informação, um $(0,31 \%)$ de Direito e um $(0,31 \%)$ respectivamente para Filosofia, Estatística, Música e Sociologia.

\section{Instrumentos}

Inventário de Resolução de Problemas Sociais (IRPS) de D'Zurilla e Nezu (1990) traduzido e adaptado para o português por Sá (2005). Trata-se de uma escala do tipo Likert de cinco pontos indo de 0 (completamente falso para mim) a 4 (completamente verdadeiro para mim). É constituído por 70 itens e caracteriza a resolução de problemas sociais como um processo complexo que envolve aspectos cognitivos, afetivos e comportamentais. O IRPS é constituído por dois fatores fundamentais: Orientação do Problema (OP; $\alpha=0,94)$ e Habilidades de Resolução de Problemas (HRP; $\alpha=0,92$ ).

Inventário de Habilidades Sociais (IHS) de Z. A. P Del Prette e A. Del Prette (2001) é um instrumento de autorrelato e mensura habilidades sociais $(\alpha=0,75)$. É constituído por 38 itens, que descrevem uma situação de relação interpessoal e uma demanda de habilidade em resposta àquela situação. A resposta ao item é em escala de cinco pontos, variando de zero (nunca ou raramente) a 4 (sempre ou quase sempre). O Inventário tem uma estrutura composta por cinco fatores: Autoafirmação e Enfrentamento com Risco (EAAR; $\alpha=0,96$ ); Autoafirmação na Expressão de Afeto Positivo (AAESP; $\alpha=0,86$ ); Conversação e Desenvoltura Social (CSD; $\alpha=0,81$ ); Autoexposição a Desconhecidos ou Situações Novas (AEDSN; $\alpha=0,75$ ) e Autocontrole da Agressividade a Situações Aversivas (ACA; $\alpha=0,74$ ).

Inventário de Estratégias de Coping (IEC) de Folkman e Lazarus (1985) adaptado para o português por Savóia, Santana e Mejias (1996). O Inventário é constituído por 66 itens (precisão total obtida pelo teste-reteste valor de $0,704)$ e envolve pensamentos e ações que as pessoas utilizam para lidar com demandas internas ou externas de um evento estressante específico. Apresenta marcação ao item em uma escala que vai de 0 (não usei esta estratégia) até 3 (usei em grande quantidade). É composto por oito fatores: Confronto (CONF), Afastamento (AFAST), Autocontrole 
(AC), Aceitação de Responsabilidade (AR), Fuga-esquiva (FE), Resolução de Problemas (RESP), Reavaliação Positiva (RP) e Suporte Social (SS).

Questionário de Vivências Acadêmicas-reduzido (QVA-r) de Almeida, Soares e Ferreira (2002) adaptado por Granado, Santos, Almeida, Soares e Guisande (2005). É composto por 55 itens em uma escala de cinco pontos, indo de 1 (nada a ver comigo) a 5 (tudo a ver comigo). $\mathrm{O}$ instrumento abrange cinco dimensões: a dimensão Pessoal é constituída de 14 itens $(\alpha=0,87)$, a Interpessoal é composta por 12 itens $(\alpha=0,86)$, a Vocacional possui 12 itens, $(\alpha=0,91)$, a Estudo- aprendizagem é constituída por nove itens $(\alpha=0,82)$ e a Institucional apresenta oito itens $(\alpha=0,71)$.

\section{Procedimentos}

Foram realizados contatos com coordenadores e docentes de diferentes instituições públicas com o objetivo de apresentar a pesquisa e os instrumentos a serem utilizados. Enfatizou-se a necessidade da realização da coleta in loco devido ao quantitativo de instrumentos a serem respondidos. Os instrumentos foram entregues na seguinte sequência: Termo de Consentimento Livre e Esclarecido, Questionário Sociodemográfico, IRPS, IHS, IEC e QVA-r. Todos os instrumentos foram respondidos em um único momento. O estudo foi submetido ao Comitê de Ética da Universidade.

\section{Análise de dados}

Utilizou-se as seguintes técnicas psicométricas para análise dos dados: Modelagem por Equações Estruturais (MEE), com o objetivo de investigar o quanto as variáveis habilidade de resolução de problemas, estratégias de coping e habilidades sociais seriam capazes de predizer os escores dos fatores do Questionário de Vivências Acadêmicas. A MEE foi realizada para a amostra total bem como para os dois grupos distintos. Os índices de ajuste utilizados na avaliação dos modelos foram o Root Mean Square Error of Aproximation (RMSEA), Comparative Fit Index (CFI) e Standardized Root-Mean-Square Residual (SRMR). Valores de RMSEA e SRMR não devem exceder 0,08, com o limite superior do seu intervalo de confiança não excedendo 0,10 . Os valores de CFI devem ser maiores que 0,90 e, preferencialmente, acima de 0,95 (Brown, 2006) e teste $t$ de Student para comparar se existia diferença significativa entre a média das amostras do estudo (estudantes cotistas e não cotistas) em relação às variáveis pesquisadas. Para todas as análises do teste $t$, foram implementados procedimentos de re-amostragem (bootstrapping;
1.000 re-amostragens, com intervalo de confiança 99\%), com vistas a apresentar maior confiabilidade aos resultados, corrigir possíveis desvio de normalidade da amostra e apresentar intervalo de confiança de $99 \%$ para as diferenças de média (Haukoos \& Lewis, 2005). Os tamanhos dos efeitos das diferenças encontradas foram avaliados por meio da estimativa $d$ de Cohen.

\section{Resultados}

As análises entre grupos foram realizadas para a amostra total de estudantes e para a amostra de cotistas e não cotistas de maneira separada. Em relação ao QVA-r, foram encontradas diferenças estatisticamente significativas apenas para o fator Pessoal $\left(2,019^{* *} p<0,001\right)$, no qual os alunos cotistas apresentaram escores menores quando comparados com a amostra de estudantes não cotistas. O tamanho de efeito foi baixo $(0,24)$. Em relação ao Inventário de Habilidades Sociais, foram encontradas diferenças estatisticamente significativas apenas para o fator Autoexposição a Desconhecidos ou Situações Novas $(2,758 * * * * p<0,001)$ para o grupo de cotistas. Este apresentou escores estatisticamente inferiores a amostra de não cotistas. Entretanto, o tamanho do efeito foi pequeno $(0,32)$.

Para o Inventário de Estratégia de Coping, foram encontradas diferenças estatisticamente significativas apenas para o fator Fuga e Esquiva $(-2,934 * * * * p<0,001)$, no qual os cotistas apresentaram escores significativamente maiores que o de IES pública no geral. O tamanho de efeito da diferença foi pequeno $(0,34)$. Em relação ao Inventário de Resolução de Problemas Sociais, não foram encontradas diferenças estatisticamente significativas. No que diz respeito ao impacto das variáveis explicativas na variável critério (QVA-r total), os índices de ajuste dos modelos se adequaram mais para o grupo de IES públicas geral quando comparados com o grupo de cotistas $\left(\Delta \chi^{2}=5,346 ; p<0,001\right)$ (amostra total de IES pública obteve-se $\chi^{2}(\mathrm{df}) 1,128$ (1); $\chi^{2} / \mathrm{dfn} 1,128$; CFI 1,000; RMSEA [90\% IC] 0,020 [0,000 - 0,152]; SRMR 0,002).

Quanto aos fatores do QVA-r, as variáveis explicaram 53,3\% $\left(\mathrm{R}^{2}=0,533\right)$ da variância do fator QVA-r Pessoal do grupo total de IES pública. A maior carga fatorial obtida foi oriunda do fator Habilidades de Resolução de Problemas do Inventário de Resolução de Problemas Sociais. No grupo de cotistas, todos os instrumentos explicaram 46,5\% $\left(R^{2}=0,465\right)$ da variância do fator QVA-r - Pessoal. A maior carga fatorial obtida foi oriunda do fator Reavaliação Positiva e Fuga e Esquiva do Inventário de Estratégias de Coping. Concernente a variância do fator QVA-r - Interpessoal, as variáveis 
explicaram $35,0 \%\left(\mathrm{R}^{2}=0,350\right)$. A maior carga fatorial obtida foi oriunda do fator Aceitação de Responsabilidade do Inventário de Estratégia de Coping. Para a amostra de estudantes cotistas, as variáveis explicaram $29,2 \%$ $\left(\mathrm{R}^{2}=0,292\right)$ da variância do fator QVA-r - Interpessoal. Em termos da variância do fator QVA-r - Carreira, as variáveis explicaram $34,6 \%\left(\mathrm{R}^{2}=0,346\right)$ e para a amostra de cotistas todas as variáveis explicaram 48,9\% $\left(\mathrm{R}^{2}=0,489\right)$ da variância do fator QVA-r - Carreira. A maior carga fatorial obtida foi oriunda do fator Suporte Social do Inventário de Estratégia de Coping para a amostra de estudantes cotistas. As variáveis do modelo proposto explicaram $52,1 \%\left(\mathrm{R}^{2}=0,521\right)$ da variância do fator QVA-r - Estudo para a amostra total e para a amostra de cotista as variáveis explicaram $51,1 \%\left(\mathrm{R}^{2}=0,511\right)$ da variância do fator QVA-r - Estudo, sendo a maior carga fatorial obtida oriunda do fator Resolução de Problema do Inventário de Estratégias de Coping. Em termos da variância do fator QVA-r - Institucional, as variáveis do modelo proposto explicaram $12,2 \%\left(R^{2}=0,122\right)$. Nenhum fator foi estatisticamente significativo na predição do QVA-r - Institucional para amostra de IES públicas no geral e para a amostra de cotistas todos os instrumentos explicaram $48,8 \%\left(\mathrm{R}^{2}=0,488\right)$ da variância do fator QVA-r Institucional. A maior carga fatorial obtida foi oriunda do fator Autoexposição a Desconhecidos ou Situações Novas do Inventário de Habilidades Sociais, seguido do fator Suporte Social do Inventário de Estratégias de Coping.

\section{Discussão}

Os resultados obtidos apontam impacto significativo para o fator Pessoal do QVA-r para os não cotistas. Tal evidência supostamente pode refletir que o estudante que ingressa nas universidades públicas, vivenciando exigências tanto acadêmicas como relacionais, forçosamente o aluno sente-se autoconfiante, capaz, satisfeito por ter alcançado a meta pretendida de ter ingressado em IES pública (Soares \& Del Prette, 2015). São resultados que contribuem com a temática, mas que ainda requerem um maior número de pesquisas. Neste seguimento, estudo realizado por Ribeiro et al. (2014), com 27 alunos de uma IES pública, de diferentes períodos e divididos em três grupos (cotista e não cotistas; não cotistas; apenas cotistas), foi concluído que em termos relacionais, há a necessidade de se discutir a convivência dos grupos fechados (cotistas e não cotistas), trazendo para análise o padrão de interação existente e pensar-se em um processo de elaboração de mudanças com o objetivo de modificar as percepções quanto aos alunos cotistas. Estes foram concebidos com menos capazes por não terem tido uma formação escolar anterior de qualidade ou mesmo advindos das camadas mais desfavorecidas da população. Contudo, a amostra contou com participantes com níveis socioeconômicos medianos e ex-alunos de escolas públicas federais conceituadas quanto a qualidade do processo de ensino e aprendizagem.

O fator Autoexposição a Desconhecidos ou Situações Novas do IHS apresentou impacto positivo para os estudantes cotistas, o que pode indicar que a universidade é um ambiente novo para o aluno e este se sente ávido para enfrentar as circunstâncias do contexto (Couto et al., 2012; Soares et al., 2016). O discente procura integrar-se por meio da exposição de ideias, do exercício da capacidade de questionar, o que nem sempre está alinhado com as ideias ou valores do interlocutor. Em outros termos, o estudante cotista procura adaptar-se acadêmica e socialmente sem receio de se expor seja para colegas e professores, o que estaria em consonância com o impacto do fator RP do IEC para o grupo de cotistas.

Ademais, as diversas situações enfrentadas e não necessariamente relacionadas as questões como autoconfiança e bem-estar podem levar o estudante a enfrentar as dificuldades da adaptação por estratégias evitativas (fator Fuga e Esquiva do IEC para os cotistas), que não são as adequadas (Oliveira et al., 2014). Karnal et al. (2017) reforçam que os programas de acesso ao Ensino Superior não são suficientes para garantirem a manutenção e conclusão da graduação, a partir do momento que o aluno administra ao longo do curso uma série de adversidades. Para os autores, fatores considerados protetivos como o apoio e o incentivo familiar contribuem para o planejamento e a determinação em finalizar o curso com qualidade de aprendizado.

Assim, os fatores que explicaram o fator Pessoal do QVA-r não diferenciaram significativamente entre os grupos. As diferenças significativas foram para Fuga e Esquiva e Reavaliação Positiva do IEC. O resultado obtido reforça a questão que diante dos desafios enfrentados no cotidiano estudantil, o aluno cotista procura interpretar as situações como experiências favoráveis. Entretanto, pode tentar fazê-lo sem os recursos internos e externos para administrar as adversidades. Matta et al. (2017) afirmam que a adaptação acadêmica ocorre quando o graduando experimenta as novas exigências inerentes à Educação Superior, como desempenho acadêmico, as normas institucionais, o convívio social e a integração. Esta etapa de vida promove questionamentos pessoais de ordem ética, vocacional, acadêmica e social e implica em novos padrões de funcionamento.

Este cenário de possibilidades e transformações requer fatores protetivos que facilitem a adaptação. Karnal (2017) em pesquisa com 13 estudantes prounistas de IES 
particular, de ambos os sexos e idade entre 19 e 40 anos, destacam que a família é percebida como fator protetivo, pelo fato do apoio afetivo e financeiro. Além de que, auxilia nos comportamentos pessoais diante de riscos de desadaptação e aponta para a existência de rede de apoio social que envolve recursos pessoais e institucionais que incentiva a enfrentar as adversidades. Um outro elemento facilitador e promotor da adaptação acadêmica é o acolhimento da IES no que concerne a informações burocráticas e de ações de assistência estudantil, tais como alimentação, transporte, inclusão digital e lazer. O que é afirmado pelos autores estaria em consonância com o impacto observado para o fator HRP do IRPS, o qual indica que o estudante busca soluções e estas devem ser planejadas e organizadas antes de serem executadas.

Neste sentido, encontra-se conformidade com o fator ACA do IHS. Nas dificuldades do dia a dia, o estudante cotista procura ser habilidoso e gerir comportamentos mais agressivos e se percebe capaz para lidar com a vida acadêmica, administrando tarefas e relacionamentos que acrescentem conhecimentos e contribuam com a futura carreira (Matta et al., 2017). Ademais, aponta para o fato de que na interação com os pares e os professores e nas atividades acadêmicas de cunho social parece que o estudante percebe dificuldades na construção de novos vínculos. Estar em uma rede que o acolha e compartilhe experiências facilitaria a integração acadêmica. Tal questão está em conformidade com o impacto obtido no fator RESP do IEC para o fator Estudo, no qual o aluno procura resolver as situações do cotidiano e talvez se perceba autoconfiante por encontrar apoio nas redes sociais construídas no ambiente acadêmico. Santos (2016); Ribeiro et al. (2014) salientam a importância de se compreender as concepções que alunos cotistas e não cotistas possuem dos benefícios proporcionados para aqueles que acessam as universidades públicas pelo sistema de cotas.

Em termos de Carreira, os resultados obtidos apontam que o estudante se percebe como confiante e capaz em estabelecer objetivos, fazer escolhas e planejar o que considera relevante para a futura profissão (Bardagi \& Boff, 2010; Santos, 2013), reforçado pelo impacto do SS do IEC. O estudante cotista procura interagir, se posicionar em relação a opiniões, estabelecer novos vínculos, trabalhar em grupo, estabelecer bons relacionamentos com docentes, construir uma nova rede de apoio como manter as que já possui e empenhar-se nas diferentes atividades. Tal impacto encontra consonância no fator EAAR do IHS, a partir do momento que ao expor e defender ideias e opiniões, mesmo que não sejam aceitas pelos interlocutores, o estudante mostra a determinação em se fazer presente socialmente e sustentar seu ponto de vista diante das situações experimentadas.
Finalmente, o impacto do fator SS do IEC sobre o fator Institucional do QVA-r pode ocorrer pelo fato de que ao identificar-se com a instituição em que estuda, conhecendo o que é oferecido e as dificuldades inerentes as exigências do ambiente acadêmico, o estudante busca suporte social para enfrentar as adversidades inerentes ao contexto, procura expor opiniões, participar de atividades e fazer novos vínculos (fator AEDSN do IHS; Sicre \& Casaro, 2014). Aparentemente, o estudante cotista confronta as adversidades inerentes do contexto acadêmico e tenta nas redes de relacionamentos superar as dificuldades e buscar apoio para seguir em frente acreditando ser capaz de se organizar academicamente e concluir com sucesso o seu curso.

Ademais, as concepções sobre as cotas geram significados específicos tanto para aqueles que se beneficiam como para os que não têm acesso. Santos (2013) aponta que os estudantes apresentam um discurso ambivalente em relação as cotas, concordam quanto a relevância de ações afirmativas que tentem corrigir um processo de exclusão existente no Ensino Superior, mas acreditam que as cotas não sejam a solução mais viável. Outro aspecto ressaltado pela autora é que há uma concepção estereotipada sobre o grupo de alunos que ascendem por meio de cotas, como se tratasse de um grupo homogêneo nas vivências. Ribeiro et al. (2014) ressaltam que estudantes cotistas tendem a idealizar as justificativas sobre a implementação de cotas quando interpelados, diferentemente dos não cotistas que concebem o benefício negativamente como gerador de preconceito, discriminação e injustiças.

\section{Considerações finais}

O estudo objetivou investigar o impacto das variáveis Coping, Habilidades Sociais, Resolução de Problemas Sociais sobre a Adaptação Acadêmica em estudantes cotistas e não cotistas das Instituições de Ensino Superior públicas. As políticas de acesso implementadas nas últimas décadas possibilitaram que discentes com uma heterogeneidade de experiências relacionais e de aprendizagem ingressassem na universidade. Muitos desses estudantes apresentam experiências anteriores que nem sempre fortalecem comportamentos que se ajustam às exigências próprias da Educação Superior.

Desta forma, as diversidades das experiências dos ingressantes na universidade conduzem a busca por mudanças tanto no âmbito pessoal, contextual e institucional. O aluno deve apresentar maior autonomia nos estudos, assim como a instituição deve contribuir com o desenvolvimento de habilidades relacionais e cognitivas e facilitar a permanência do estudante por meio, por exemplo, da flexibilidade curricular (Mofidi et al., 2014). 
O impacto alcançado pelas variáveis do modelo proposto, indicam a relevância das relações interpessoais como o suporte que provavelmente assegura a integração do estudante, a permanência e continuidade da formação com aprimoramento da aprendizagem. Ademais, é mister conhecer os recursos internos e as estratégias utilizadas pelos estudantes para enfrentarem as dificuldades e o empenho cognitivo do aluno cotista para adequar-se à universidade, enfrentando os desafios com estratégias de Suporte Social, de Resolução de Problemas, expondo-se a situações Novas e a Desconhecidos como sendo relevantes para a adaptação ao Ensino Superior.
O aluno beneficiário do sistema de cotas confronta os impasses inerentes à realidade universitária provavelmente utilizando estratégias de enfrentamento inadequadas, o que não contribui para a sua saúde mental (Oliveira et al., 2014) e não proporciona o desempenho esperado (Araújo, 2017). Neste sentido, estudos prospectivos podem investigar as diferenças de estratégias de coping e habilidades sociais utilizadas por alunos e alunas cotistas, assim como a introdução de outras variáveis em modelo teórico, considerando que a adaptação acadêmica é um fenômeno multideterminado.

\section{Referências}

Almeida, L. S. (2007). Transição, adaptação académica e êxito escolar no ensino superior. Revista Galego-Portuguesa de Psicoloxía e Educación, 14, 203-215.

Almeida, L.S., Soares, A.P.C., \& Ferreira, J.A. (2002). Questionário de Vivências Acadêmicas (QVA-r): Avaliação do ajustamento dos estudantes universitários. Avaliação Psicológica, 2, 81-9.

Antoniazzi, A., Dell'Aglio, D., \& Bandeira, D. (1998). O conceito de coping: uma revisão teórica. Estudos de Psicologia, 3(2), 273-294.

Araújo, M. A. (2017). Sucesso no Ensino Superior: Uma revisão e conceptualização. Revista de Estudios e Investigación en Psicología y Educación, 4(2), 132-141.

Associação Brasileira de Empresas de Pesquisa (2015). Critério Brasil. Recuperado em 02 maio 2015, de http://www. abep.org

Bardagi, M. P., Boff, R. M. (2010). Autoconceito, autoeficácia profissional e comportamento exploratório em universitários concluintes. Avaliação: Revista da Avaliação da Educação Superior (Campinas), 15, 41-56.

Brown, T. A. (2006). Confirmatory factor analysis for applied research. New York: The Guilford Press.

Castro, R. V., \& Almeida L. S. (2016). Ser estudante no ensino superior: Observatório dos percursos académicos dos estudantes da UMinho. In: L.S. Almeida \& R. V. Castro (Orgs.) (pp. 15-31), Ser estudante no Ensino Superior: O caso dos estudantes do $1^{\circ}$ ano. Portugal, Braga: Universidade do Minho.

Cardoso, C. B. (2008). Efeitos da política de cotas na Universidade de Brasília: uma análise do rendimento e da evasão. (Dissertação de mestrado em Educação). Universidade de Brasília, Brasília, Faculdade de Educação.

Couto, G., Vandenberghe, L., Tavares, W. M., \& Silva, R. L. F. C. (2012). Interações e habilidades sociais entre universitários: um estudo correlacional. Estudos de Psicologia (Campinas), 29, 667-677.

Del Prette, A., \& Del Prette, Z. A. P. (Orgs.). (2017). Habilidades sociais intervenções efetivas em grupo (2a. ed.). São Paulo: Pearson.

Del Prette, Z. A. P., \& Del Prette, A. (2001). Inventário de habilidades sociais: manual de aplicação, apuração e interpretação. São Paulo: Casa do Psicólogo.

D'Zurilla, T. J., \& Nezu, A. M. (1990). Development and preliminary evaluation of the Social Problem-Solving Inventory (SPSI). Psychological Assessment: A Journal of Consulting and Clinical Psychology, 2, 156-163.

D’Zurilla, T. J., Nezu, A. M., \& Maydeu-Olivares, A. (2002). Social Problem-Solving Inventory. North Tonawanda: Multi-Health Systems.

D’Zurilla, T. J., \& Sheedy, C. F. (1992). The Relation Between Social Problem-Solving Ability and Subsequent Level of Academic Competence in College Students. Cognitive Therapy and Research, 16(5), 589-599.

Fang, J., Luo, Y., Li, Y., \& Huang, W. (2016). Social problem-solving in Chinese baccalaureate nursing students. Journal of Evidence-Based Medicine, 9, 181-187. doi: 0.1111/jebm.12232 Folkman, S., \& Lazurus, R. S. (1985). If it changes it must be a process: study of emotion and coping during three stages of a college examination. Journal of Personality and Social Psychology, 48(1), 150-170.

Granado, J. I. F., Santos, A. A., Almeida, L. S., Soares, A. P., \& Guisande, M. A. (2005). Integração acadêmica de estudantes universitários: contributos para a adaptação e validação do QVA- r no Brasil. Psicologia e Educação, IV (1), 33-43.

Haukoos, J. S., \& Lewis, R. J. (2005). Advanced statistics: Bootstrapping confidence intervals for statistics with "difficult" distributions. Academic Emergency Medicine, 12(4), 360-365. doi: 10.1197/j.aem.2004.11.018

Iturra, G. O., Goic, C. E., Astete, E. P., \& Jara, M. O. (2012). Habilidades sociales y rendimento académico: Una mirada desde el género. Acta Colombiana de Psicología, 15(2), 21-28. 
Lau, Y. (2014). Factors affecting the social problem-solving ability of baccalaureate nursing students. Nurse Education Today, 34, 121-126.

Lazarus, R. S., \& Folkman, S. (1984). Stress, appraisal, and coping. New York: Springer.

Matta, C. M. B., Lebrão, S. M. G., \& Heleno, M. G. V. (2017). Adaptação, rendimento, evasão e vivências acadêmicas no ensino superior: revisão da literatura. Psicologia Escolar e Educacional, 21(3), 583-591. doi: $10.1590 / 2175-3539 / 2017 / 0213111118$

Mello, T. V. S., \& Soares, A. B. (2014). Habilidades sociales y frustración en estudiantes de medicina. Ciencias Psicológicas, VIII (2), 163-172.

Mofidi, T., El-Alayli, A., \& Brown, A. A. (2014). Trait gratitude and grateful coping asthey relate to college student persistence, success, and integration in school. Journal of College Student Retention, 16(3) 325-349. doi: /10.2190/ CS.16.3.b

Moreno, P. F., \& Soares, a. B. (2014). O que vai acontecer quando eu estiver na universidade? Expectativas de jovens estudantes brasileiros. Aletheia, 45, 114-127.

Oliveira, C. T, Carlotto, R. C., Vasconcelos, S. J. L., \& Dias, A. C. G. (2014). Adaptação acadêmica e coping em estudantes universitários brasileiros: Uma revisão de literatura. Revista Brasileira de Orientação Profissional, 15(2), 177-186.

Organização de Cooperação e Desenvolvimento Econômico-OCDE (2012). OECD Higher Education Programme IMHE General Conference. França, Paris: Creative Connection.

Plano Nacional de Educação 2014-2024 (2014). Recuperado em 20 Set. 2014, de http://www.observatoriodopne.org.br/ uploads/reference/file/439/documento-referencia.pdf.

Ribeiro, E. M. B. A., Amaral, L. L. L., Peixoto, A. L. A., Oliveira, L. M. G., \& Bastos, A. V. B. (2014). A convivência entre estudantes cotistas e não cotistas na Universidade Federal da Bahia. In XIV colóquio internacional de gestão universitária - CIGU, Florianópolis, 3-5 dez., Instituto de Pesquisas e Estudos em Administração UniversitáriaINPEAU. Santa Catarina, 1-14.

Sá, A. P. (2005, 15 de abril). A Importância do Psicodiagnóstico Diferencial dos Transtornos de Personalidade no Tratamento dos Transtornos de Ansiedade. Trabalho apresentado no V Congresso da Sociedade Brasileira de Terapias Cognitivas. Resumo recuperado em 12 junho 2016, de http://cbtc.fbtc.org.br/\#/ficha/2005

Santos, P. V. S. (2013). Adaptação à Universidade dos Estudantes Cotistas e Não Cotistas: Relação entre Vivência Acadêmica e Intenção de Evasão. (Dissertação de mestrado em Educação). Faculdade de Psicologia, Universidade Federal da Bahia, Salvador.

Savóia, M. G., Santana, P.R., \& Mejias, N. P. (1996). Adaptação do inventário de estratégias de coping de Folkman e Lazarus para o português. Psicologia USP, 7(1/2), 183-201.

Schofield, J. W., Hausmann, L. R. M., Ye, F., \& Woods, R. L. (2010). Intergroup friendships on campus: Predicting close and casual friendships between White and African American first- year college students. Group Processes \& Intergroup Relations, 13(5), 585-602. doi: 10.1177/1368430210362437

Sicre, E., \& Casaro, L. (2014). Estrategias de afrontamiento en estudiantes de Psicología. Revista de Psicología, 10(20), 61-72.

Soares, A. B. \& Del Prette, Z. A. P. (2015). Habilidades sociais e adaptação à universidade: Convergências e divergências dos construtos. Análise Psicológica, 2(XXXIII), 139- 151.doi: 10.14417/ap.911

Soares, A. B., Gomes, G., Maia, F. A., Gomes, C. A. O., \& Monteiro, M. C. (2016). Relações interpessoais na universidade: o que pensam estudantes da graduação em psicologia? Estudos Interdisciplinares em Psicologia, 7(1), 56-76.

Soares, A. B, Poubel, L. N., \& Melo, T.V.S. (2009). Habilidades sociais e adaptação acadêmica: um estudo comparativo em instituições de ensino público e privado. Aletheia, 29, 27-42.

Souza, L. K., Lourenço E., \& Santos, M. R. G. (2016). Adaptação à universidade em estudantes ingressantes na graduação em psicologia. Psicologia da Educação, 42(1), 35-40. doi: 10.5935/2175-3520.20150023

Tinto, V. (1975). Dropout from higher education: A theoretical synthesis of recent research. Review of Educational Research, 45, 89-125. doi: 10.3102/00346543045001089

Recebido 28/01/2018

$1^{a}$ Reformulação 03/06/2018

$2^{a}$ Reformulação 06/08/2018

Aceite Final 07/10/2018

Sobre as autoras

Marcia Cristina Monteiro é psicóloga, Doutora em Psicologia Social pela Universidade Salgado de Oliveira (Universo), Mestre em Psicologia social e da Personalidade pela Universidade Federal do Rio de Janeiro e professora de Psicologia na Universidade Brasil (UNIESP).

Adriana Benevides Soares é psicóloga, Pós-doutora pela Universidade Federal de São Carlos, Doutora em Psicologia Cognitiva pela Universidade de Paris XI, professora do Programa de Pós-graduação em Psicologia da UNIVERSO e da Universidade do Estado do Rio de Janeiro. 